\title{
Synthesis of Alkylated Aminofluorenes by Palladium-Catalyzed Substitution at Halofluorenes
}

\author{
Ginagunta Saroja, Zhang Pingzhu, Nikolaus P. Ernsting, Jürgen Liebscher* \\ Department of Chemistry, Humboldt-University, Brook Taylor-Str. 2, \\ 12489 Berlin, Germany

\section{liebscher@chemie.hu-berlin.de}


Table of contents:

General Experimental..................................S3

9H-Fluorene-2-carbonitrile..............................S3

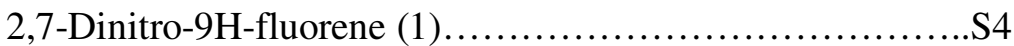

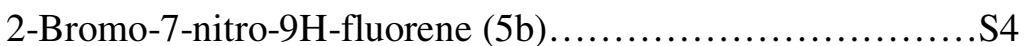

7-Bromo-9H-fluorene-2-carbonitrile (5c)..................S4

9,9-Dialkyl-9H-fluorenes 7, General Procedure...............S5

2-Iodo-7-nitro-9,9-dipropyl-9H-fluorene (7a)...............S5

2-Bromo-7-nitro-9,9-dipropyl-9H-fluorene (7b).............S5

2-Bromo-9,9-dimethyl-7-nitro-9H-fluorene (7c).............S5

2-Bromo-7-cyano-9,9-dimethyl-9H-fluorene (7d)............S6

2-Bromo-9,9-diethyl-9H-fluorene (7e).....................S6

2,7-Dibromo-9,9-diethyl-9H-fluorene (7f) .................S6 
General Experimental: Chemicals including 2-nitrofluorene, 2-bromofluorene 5d, 2,7dinitrofluorene and 2-formylfluorene were purchased. 2-Iodo-7-nitro-9H-fluorene $\mathbf{5 a},{ }^{1}$ and 2,7-dibromofluorene $\mathbf{5} \mathbf{e}^{2}$ were prepared according to the literature. 2-Cyanofluorene and 2amino-7-nitrofluorene are known but more efficient preparations were developed (see supporting information). TLC analysis was performed on silica gel $60 \mathrm{~F}_{254}$ plates and visualized with UV illumination and charring with phosphomolybdic acid in EtOH (5\%, v/v) or $0.3 \%$ ninhydrine in EtOH. Column chromatography was conducted with silica gel 60 (400639 mesh). Melting points were determined on a hot-stage apparatus and are reported uncorrected. ${ }^{1} \mathrm{H}$ NMR and ${ }^{13} \mathrm{C}$ NMR spectra were recorded at 300 and $75.5 \mathrm{MHz}$, respectively in $\mathrm{CDCl}_{3}$ with TMS as internal standard. Mass spectra were measured at $70 \mathrm{eV}$.

9H-Fluorene-2-carbonitrile: To the solution of $9 H$-fluorene-2-carbaldehyde ( $3 \mathrm{~g}, 0.015 \mathrm{~mol})$ in $\mathrm{N}$-methylpyrrolidone $(23 \mathrm{~mL}), \mathrm{H}_{2} \mathrm{NOH} \cdot \mathrm{HCl}(1.25 \mathrm{~g}, 0.018 \mathrm{~mol})$ was added. After heating to $115^{\circ} \mathrm{C}$ for $5-6 \mathrm{~h}$, the reaction mixture was poured into cold water. The light brownish precipitate formed was collected by filtration; it was subjected to recrystallisation from ethanol, water mixture to yield $2.7 \mathrm{~g}$ (94\% yield). mp $87{ }^{\circ} \mathrm{C},{ }^{1} \mathrm{H} \mathrm{NMR}\left(300 \mathrm{MHz}, \mathrm{CDCl}_{3}\right)$ : $\delta$ $7.82(\mathrm{~s}, 1 \mathrm{H}, \underline{\mathrm{CH}}), 7.79$ (s, 1H, $\underline{\mathrm{CH}}), 7.66-7.63(\mathrm{dm}, 1 \mathrm{H}, \underline{\mathrm{CH}}), 7.59-7.56(\mathrm{dm}, 1 \mathrm{H}, \underline{\mathrm{CH}}), 7.42-$ $7.38(\mathrm{~m}, 2 \mathrm{H}, \underline{\mathrm{CH}}), 3.91\left(\mathrm{~s}, 2 \mathrm{H}, \underline{\mathrm{CH}}_{2}\right) .{ }^{13} \mathrm{C} \mathrm{NMR}\left(300 \mathrm{MHz}, \mathrm{CDCl}_{3}\right): \delta 146.2,143.8,139.8$, 131.1, 128.6, 127.2, 125.3, 120.9, 120.4, 119.6, 109.6, 36.7. Anal calc for $\mathrm{C}_{14} \mathrm{H}_{9} \mathrm{~N}$ : C, 87.93; H, 4.74; N, 7.32. Found: C, 87.14; H, 5.02; N, 7.51.

\footnotetext{
${ }^{1}$ Marhevko, V. C.; Ebner, N. A.; Sehorn, R. D, Hanna, P. E. J. Med. Chem. 1985, 28, 18.

${ }^{2}$ Kelley, Charles J.; Ghiorghis, Alem; Kauffman, Joel M. J.Chem.Res.(Miniprint) 1997, 12, 2701.
}

2,7-Dinitro-9H-fluorene (1): Fuming $\mathrm{HNO}_{3}(90 \mathrm{~mL})$ was slowly added to mixture of 2nitrofluorene $(9 \mathrm{~g}, 0.043 \mathrm{~mol})$ and acetic acid $(90 \mathrm{~mL})$ under vigorous stirring and careful cooling with an ice bath. After stirring for $5 \mathrm{~h}$ at room temperature, the yellow product 
separated. It was filtered off,, washed with water and dried to yield crude 1 (6.93 g, 62\% yield). mp 295-298 ${ }^{\circ} \mathrm{C}$, Anal calc for $\mathrm{C}_{13} \mathrm{H}_{8} \mathrm{~N}_{2} \mathrm{O}_{2}: \mathrm{C}, 60.93 ; \mathrm{H}, 3.31 ; \mathrm{N}, 10.94$. Found: $\mathrm{C}$, 61,$29 ; \mathrm{H}, 3.28 ; \mathrm{N}, 10.68$.

2-Bromo-7-nitro-9H-fluorene (5b): To a stirred solution of 2-nitrofluorene (8 g, $0.038 \mathrm{~mol})$ in dry $\mathrm{CH}_{2} \mathrm{Cl}_{2}$ ( $40 \mathrm{~mL}$ ) was added $\mathrm{Br}_{2}$ ( $\left.0.076 \mathrm{~mol}\right)$. The $\mathrm{HBr}$, which soon evolved from solution was guided through a trap to a scrubbing solution of $\mathrm{NaOH}$. The mixture was stirred for 5-6 h. A yellow precipitate appeared, which was filtered off, washed with $5 \% \mathrm{NaHSO}_{3}$ and water and dried with $\mathrm{MgSO}_{4}$. The crude yellowish product was purified from recrystallisation from a mixture of DMF and water to yield light yellow material $\mathbf{5 b}(7.7 \mathrm{~g}$, $70 \%$ yield). M. p. $235-237{ }^{\circ} \mathrm{C},{ }^{1} \mathrm{H}$ NMR (300 MHz, DMF- $\left.d \sigma\right): \delta 8.50$ (s, 1H), 8.33 (dd, 1H), $8.22(\mathrm{~d}, 1 \mathrm{H}), 8.09(\mathrm{dd}, 1 \mathrm{H}), 7.94(\mathrm{~s}, 1 \mathrm{H}), 7.69(\mathrm{dd}, 1 \mathrm{H}), 4.18(\mathrm{~s}, 2 \mathrm{H})$. Anal Calcd for $\mathrm{C}_{13} \mathrm{H}_{8} \mathrm{BrNO}_{2}$ : C, 53.82; H, 2.78; N, 4.83. Found: C, 53.72; H, 2.83; N, 4.90..

7-Bromo-9H-fluorene-2-carbonitrile (5c): To a stirred solution of 9H-fluorene-2carbonitrile (500 mg, $2.6 \mathrm{mmol})$ in dry $\mathrm{CH}_{2} \mathrm{Cl}_{2}(6 \mathrm{~mL})$ was added $\mathrm{Br}_{2}(10.5 \mathrm{mmol})$. The $\mathrm{HBr}$ evolved from solution was guided through a trap to a scrubbing solution of $\mathrm{NaOH}$. The mixture was stirring for 5-6 h. The yellow precipitate was filtered off, washed with 5\% $\mathrm{NaHSO}_{3}$ and water and was dried. The crude yellowish solid was purified by recrystallisation from DMF/water mixture to yield yellow product 5c (380 mg, $70 \%$ yield). M.p. $172^{\circ} \mathrm{C}$ Anal calc for $\mathrm{C}_{14} \mathrm{H}_{8} \mathrm{BrN}$ : C, 62.25; H, 2.99; N, 5.19. Found: C, 62.31; H, 3.12; N, 5.38. ${ }^{1} \mathrm{H}$ $\left(300 \mathrm{MHz} \mathrm{CDCl}_{3}\right): \delta 3.90\left(\mathrm{~s}, 2 \mathrm{H}, \mathrm{CH}_{2}\right), 7.79(\mathrm{~d}, J=7.5 \mathrm{~Hz}, 2 \mathrm{H}, \mathrm{CH}), 7.54(\mathrm{dd}, J=8.3 \mathrm{~Hz}$, $1 \mathrm{H}, \mathrm{CH}), 7.65(\mathrm{~d}, J=8.3 \mathrm{~Hz}, 2 \mathrm{H}, \mathrm{CH}), 7.7(\mathrm{~s}, 1 \mathrm{H}, \mathrm{CH}) .{ }^{13} \mathrm{C} \mathrm{NMR}\left(300 \mathrm{MHz} \mathrm{CDCl}_{3}\right): \delta 36.55$ $\left(\mathrm{CH}_{2}\right), 110.11(\mathrm{C}), 119.41(\mathrm{C}), 120.44(\mathrm{CH}), 122.13(\mathrm{CH}), 122.63(\mathrm{C}), 128.55(\mathrm{CH}), 130.53$ $(\mathrm{CH}), 131.33(\mathrm{CH}), 138.81(\mathrm{C}), 143.20(\mathrm{C}), 145.08(\mathrm{C}), 145.70(\mathrm{C})$. Anal. Calcd for $\mathrm{C}_{14} \mathrm{H}_{8} \mathrm{BrN}$ : C, 62.25; H, 2.99; N, 5.19. Found: C, 62.31; H, 3.12; N, 5.38.

S4

9,9-Dialkyl-9H-fluorenes 7, General Procedure: A mixture of the fluorene 5 (1 mmol), alkyl halide (2.5 mmol, MeI or EtI or PrBr), KI (0.12 mmol) and DMSO $82.2 \mathrm{~mL})$ was stirred at room temperature under argon and powdered $\mathrm{KOH}$ or t-BuOK (4.5 mmol) was slowly 
added. The color of the mixture turned from orange-red to dark green or purple. After stirring for 3-5 $\mathrm{h}$, the reaction mixture was poured into water $(150 \mathrm{~mL})$. The product was filtered off and dried.

2-Iodo-7-nitro-9,9-dipropyl-9H-fluorene (7a): Yield 96\%, dark yellow powder, mp 105$110^{\circ} \mathrm{C}^{1} \mathrm{H}\left(300 \mathrm{MHz} \mathrm{CDCl}_{3}\right): \delta 0.68\left(\mathrm{~m}, 10 \mathrm{H}, \mathrm{CH}_{2}, \mathrm{CH}_{3}\right), 1.99\left(\mathrm{~m}, 4 \mathrm{H}, \mathrm{CH}_{2}\right), 7.52(\mathrm{~d}, J=7.9$ $\mathrm{Hz}, 1 \mathrm{H}, \mathrm{CH}), 7.75$ (t, $J=8.7 \mathrm{~Hz}, 3 \mathrm{H}, \mathrm{CH}), 8.20$ (d, $J=1.9 \mathrm{~Hz}, 1 \mathrm{H}, \mathrm{CH}), 8.25(\mathrm{dd}, J=8.7 \mathrm{~Hz}$, 1H, CH). ${ }^{13} \mathrm{C}$ NMR (300MHz $\left.\mathrm{CDCl}_{3}\right): \delta 14.2\left(\mathrm{CH}_{3}\right), 17.1\left(\mathrm{CH}_{2}\right), 42.2\left(\mathrm{CH}_{2}\right), 56.1(\mathrm{C}), 95.5$ (C), $118.3(\mathrm{CH}), 120.0(\mathrm{CH}), 122.7(\mathrm{CH}), 123.4(\mathrm{CH}), 132.5(\mathrm{CH}), 136.6(\mathrm{CH}), 146.5(\mathrm{C})$, 147.5 (C), $151.4(\mathrm{C}), 154.4$ (C). Anal. calc for $\mathrm{C}_{19} \mathrm{H}_{20} \mathrm{INO}_{2}$ : C, 54.17; H, 4.79; I, 30.12; N, 3.32; O, 7.60. Found: C,54.33; H, 4.19; N, 3.55.

2-Bromo-7-nitro-9,9-dipropyl-9H-fluorene (7b): yield 79\% (using $\mathrm{Pr}-\mathrm{Br}$ ), light brown solid, mp $109-115^{\circ} \mathrm{C},{ }^{1} \mathrm{H}\left(300 \mathrm{MHz}_{\mathrm{CDCl}}\right)$ ) $\delta 0.61\left(\mathrm{~m}, 10 \mathrm{H}, \mathrm{CH}_{2}, \mathrm{CH}_{3}\right), 1.92\left(\mathrm{~m}, 4 \mathrm{H}, \mathrm{CH}_{2}\right)$, 7.46 (m. 2H, CH), 7.57 (d, J=7.5 Hz, 1H), CH), 7.69 (d, $J=8.3 \mathrm{~Hz}, 1 \mathrm{H}, \mathrm{CH}), 8.12$ (d, $J=$ $1.9 \mathrm{~Hz}, 1 \mathrm{H}, \mathrm{CH}), 8.18(\mathrm{dd}, J=8.3 \mathrm{~Hz}, 1 \mathrm{H}, \mathrm{CH}) .{ }^{13} \mathrm{C} \mathrm{NMR}\left(300 \mathrm{MHz} \mathrm{CDCl}_{3}\right): \delta 14.2\left(\mathrm{CH}_{3}\right)$, $17.1\left(\mathrm{CH}_{2}\right), 42.2\left(\mathrm{CH}_{2}\right), 56.1(\mathrm{C}), 118.3(\mathrm{CH}), 119.9(\mathrm{CH}), 122.4(\mathrm{CH}), 123.4(\mathrm{CH}) 123.69$ (C), $126.6(\mathrm{CH}), 130.7(\mathrm{CH}), 137.7(\mathrm{C}), 146.4(\mathrm{C}), 147.4(\mathrm{C}), 151.6(\mathrm{C}), 154.4(\mathrm{C})$. Anal. Calcd for $\mathrm{C}_{19} \mathrm{H}_{20} \mathrm{BrNO}_{2}$ : C, 60.97; H, 5.39; Br, 21.35; N, 3.74; O, 8.55. Found: C, 60.87; H, 5.50; Br, 20.47; N, 3.89 .

2-Bromo-9,9-dimethyl-7-nitro-9H-fluorene (7c): Yield 91\%, yellowish solid, mp 225$242^{\circ} \mathrm{C} ;{ }^{1} \mathrm{H}\left(300 \mathrm{MHz} \mathrm{CDCl}_{3}\right): \delta 1.53\left(\mathrm{~s}, 6 \mathrm{H}, \mathrm{CH}_{3}\right), 7.54(\mathrm{dd} . J=8.3 \mathrm{~Hz}, 1 \mathrm{H}, \mathrm{CH}), 7.63(\mathrm{~d}, J=$ $1.5 \mathrm{~Hz}, 1 \mathrm{H}, \mathrm{CH}), 7.66(\mathrm{~d}, J=8.3 \mathrm{~Hz}, 1 \mathrm{H}, \mathrm{CH}), 7.79(\mathrm{dd}, J=8.3 \mathrm{~Hz}, 1 \mathrm{H}, \mathrm{CH}), 8.26$ (m, 2H, $\mathrm{CH}) ;{ }^{13} \mathrm{C}$ NMR (300MHz, $\left.\mathrm{CDCl}_{3}\right): \delta 26.6\left(\mathrm{CH}_{3}\right), 47.6(\mathrm{C}), 118.4(\mathrm{CH}), 120.3(\mathrm{CH}), 122.8$ $(\mathrm{CH}), 123.5(\mathrm{CH}), 126.6(\mathrm{CH}), 130.9(\mathrm{CH}), 135.8(\mathrm{C}), 144.6(\mathrm{C}), 147.4(\mathrm{C}), 154.2(\mathrm{C}), 156.9$ (C). Anal.Calcd for $\mathrm{C}_{15} \mathrm{H}_{12} \mathrm{BrNO}_{2}$ : C, 56.62; H, 3.80; Br, 25.11; N, 4.40; O, 10.06. Found: C, $56.62 ; \mathrm{H}, 3.87 ; \mathrm{N}, 4.48$.

S5

2-Bromo-7-cyano-9,9-dimethyl-9H-fluorene (7d): Yield : 78\%, colorless crystals, mp 180$190^{\circ} \mathrm{C} ;{ }^{1} \mathrm{H}\left(300 \mathrm{MHz} \mathrm{CDCl}_{3}\right): \delta 1.49\left(\mathrm{~s}, 6 \mathrm{H}, \mathrm{CH}_{3}\right), 7.52(\mathrm{~d}, J=8.3 \mathrm{~Hz}, 1 \mathrm{H}, \mathrm{CH}), 7.62(\mathrm{~d}, 2 \mathrm{H}, J$ $=8.3 \mathrm{~Hz}, 2 \mathrm{H}, \mathrm{CH}), 7.67(\mathrm{~d}, J=8.3 \mathrm{~Hz}, 2 \mathrm{H}, \mathrm{CH}), 7.75(\mathrm{~d}, J=7.9 \mathrm{~Hz}, 1 \mathrm{H}, \mathrm{CH}) ;{ }^{13} \mathrm{C} \mathrm{NMR}$ 
(300MHz $\left.\mathrm{CDCl}_{3}\right): \delta 26.7\left(\mathrm{CH}_{3}\right), 47.4(\mathrm{C}), 110.6(\mathrm{C}), 119.5(\mathrm{C}), 120.6(\mathrm{CH}), 122.4(\mathrm{CH})$, $123.2(\mathrm{C}), 126.5(\mathrm{CH}), 130.7$ (C), $131.6(\mathrm{CH}), 136.2$ (C), 142.7 (C), 153.7 (C), 156.1 (C); Anal. Calcd for $\mathrm{C}_{16} \mathrm{H}_{12} \mathrm{BrN}$ : C, 64.45; H, 4.06; Br, 26.80; N, 4.70. Found: C, 64.24; H, 4.31; $\mathrm{N}, 4.80$.

2-Bromo-9,9-diethyl-9H-fluorene (7e): Yield: $86 \%$, orange solid, mp $40-42{ }^{\circ} \mathrm{C} ;{ }^{1} \mathrm{H}(300 \mathrm{MHz}$ $\left.\mathrm{CDCl}_{3}\right): \delta 0.31\left(\mathrm{t}, J=7.5 \mathrm{~Hz}, 6 \mathrm{H}, \mathrm{CH}_{3}\right), 2.00\left(\mathrm{q}, J=7.5 \mathrm{~Hz}, 4 \mathrm{H}, \mathrm{CH}_{2}\right), 7.31(\mathrm{~m}, 3 \mathrm{H}, \mathrm{CH}), 7.45$ $(\mathrm{dd}, J=6.4 \mathrm{~Hz}, 2 \mathrm{H}, \mathrm{CH}), 7.56(\mathrm{dd}, J=6.4 \mathrm{~Hz}, 1 \mathrm{H}, \mathrm{CH}), 7.66(\mathrm{~m}, J=3.4 \mathrm{~Hz}, 1 \mathrm{H}, \mathrm{CH}) ;{ }^{13} \mathrm{C}$ NMR (300MHz $\left.\mathrm{CDCl}_{3}\right): \delta 8.4\left(\mathrm{CH}_{3}\right), 32.7\left(\mathrm{CH}_{2}\right), 56.0(\mathrm{C}), 119.7(\mathrm{CH}), 121.0(\mathrm{CH}), 122.9$ $(\mathrm{CH}), 126.2(\mathrm{CH}), 127.0(\mathrm{CH}), 127.5(\mathrm{CH}), 129.9(\mathrm{CH}), 140.4(\mathrm{C}), 140.5(\mathrm{C}), 149.4(\mathrm{C})$, 152.1 (C); Anal. Calcd for $\mathrm{C}_{17} \mathrm{H}_{17} \mathrm{Br}$ : C, 67.78; H, 5.69; Br, 26.53. Found: C, 67.02; H, 5.87.

2,7-Dibromo-9,9-diethyl-9H-fluorene (7f): Yield 97\%, colorless crystals $164-170^{\circ} \mathrm{C}$;

${ }^{1} \mathrm{H}\left(300 \mathrm{MHz} \mathrm{CDCl}_{3}\right): \delta 1.44\left(\mathrm{~s}, 6 \mathrm{H}, \mathrm{CH}_{3}\right), 7.44(\mathrm{dd}, J=8.3 \mathrm{~Hz}, 2 \mathrm{H}, \mathrm{CH}), 7.53(\mathrm{~d}, J=8.7 \mathrm{~Hz}$, $4 \mathrm{H}, \mathrm{CH}) ;{ }^{13} \mathrm{C}$ NMR $\left(300 \mathrm{MHz} \mathrm{CDCl}_{3}\right): \delta 26.8\left(\mathrm{CH}_{3}\right), 47.3(\mathrm{C}), 121.4(\mathrm{CH}), 126.2(\mathrm{CH}), 130.3$ $(\mathrm{CH}), 137.1(\mathrm{C}), 155.2(\mathrm{C})$; Anal. Calcd for $\mathrm{C}_{15} \mathrm{H}_{12} \mathrm{Br}_{2}$ : C, 51.17; H, 3.44; $\mathrm{Br}, 45.39$. Found: C, 50.94; H, 3.70. 